\title{
Educational Tool for Testing Emotion Recognition Abilities in Adolescents
}

\author{
Cristina Costescu ${ }^{1}$, Adrian Rosan ${ }^{1}$, Andrea Hathazi ${ }^{1}$, Marian \\ Pădure $^{1}$, Nagy Brigitta ${ }^{1}$, Attila Kovari ${ }^{2}$, Jozsef Katona ${ }^{2}$, Serge \\ Thill $^{3}$, Ilona Heldal ${ }^{4}$ \\ ${ }^{1}$ Babes-Bolyai University, Faculty of Psychology and Educational Sciences \\ 7 Sindicatelor Street, RO-400029, Cluj-Napoca, Romania \\ E-mail: $\{$ cristina.costescu $\} @$ ubbcluj.ro \\ ${ }^{2}$ University of Dunaujvaros, CogInfoCom Based LearnAbility Research Team \\ Tancsics M. 1/A, 2400 Dunaujvaros, Hungary \\ E-mail: \{kovari, katonaj\}@ uniduna.hu \\ ${ }^{3}$ Donders Centre for Cognition Donders Institute for Brain, Cognition, and \\ BehaviourRadboud University Nijmegen, Netherland \\ E-mail: \{s.thill\}@donders.ru.nl \\ ${ }^{4}$ Western Norway University, Department of Computing, Mathematics, and \\ Physics of Applied Sciences, Bergen, Norway \\ E-mail: \{ilona.heldal\}@hvl.no
}

\begin{abstract}
Emotion recognition represents an important predictor for the development of social interactions. Previous studies have shown that the ability to recognize emotions, the speed, and accuracy, with which individuals process emotions, is an ability that develops with age, starting from early childhood. Children with autism spectrum disorder (ASD) show reduced attention to faces and they have difficulties in identifying emotions. Our goal was to investigate the effectiveness of a technology-based educational tool for assessing emotion recognition skills in individuals with ASD and typical developing children.51 children aged between 12 and 14 years were enrolled in our study, out of which 11 have a diagnosis of ASD. Our results reveal that adolescents perceive emotions differently depending on the type of stimuli that we use and on their ages.
\end{abstract}

Keywords: emotion recognition; educational tool; adolescents; autism spectrum disorder

\section{Introduction}

Socio-emotional development of adolescents is highly connected with initiating social interactions and maintaining friendships. The ability to recognize facial expressions is part of social cognition development, which includes the 
understanding of other's intentions [1-2]. Labeling basic emotions, such as: happiness, sadness, fear, and anger, is a task that preschoolers can do it easily; moreover, by the age of four years, they start recognizing more complex emotions [3]. There are a lot of variables that can influence the ability of emotion recognition, but recent studies suggest that the ability to recognize facial expressions can be related to the type of stimuli/tasks that are being used in the assessment process [4-5]. The ability to recognize emotions appears to be a continuous process, meaning that the performance of correctly identifying facial expressions improves from childhood to adulthood [6-7]. When it comes to atypical development the ability to recognize emotion can be impaired and it can interfere with children's interpersonal connections and socializing. [8-9].

Autism spectrum disorder (ASD) is a neurodevelopmental condition that affects social interaction, communication, interests, and behavior. There are some studies showing that individuals with ASD have difficulties in accurately identifying emotions and interpreting socio-emotional cues [10-11]. According to recent studies, the ability to identify facial expressions is considered part of social communication skills and can predict adaptive social behavior [12].

While in typical developing children the capacity to distinguish between different types of emotions develops in their infancy [13-14] and children become more and better at this as they grow, in contrast, individuals with ASD demonstrate decreased interest and attention [15-17]. Therefore. one possible explanation for the facial expression recognition difficulties in children with ASD is the fact that their attention to faces, more exactly to eyes, is impaired [18-19]. Moreover, recent research revealed that children with ASD process faces gradually (i.e. piece by piece) rather than holistically [20-21]. Considering all the above research and also other important studies in this domain we may state that children with ASD experience deficits in facial emotion recognition [22-25]. However, there are a couple of studies that revealed interesting results in this domain, considering that children with ASD may have similar performances in identifying emotions as typically developing children [26-29].

These emotion recognition difficulties are important to be investigated because they can impact the social development of individuals with ASD and lead to social exclusion and can represent an obstacle in accessing and sustaining education [3031]. Recent approaches suggest that the use of technology as educational instruments for evaluating and improving the emotional abilities of children with ASD may be particularly beneficial for addressing motivation and generalization issues. The rationale of using technological tools in the educational process of children with ASD is that these types of tools combine learning theory, motivational theory and game design to create a unique and exciting remediation ways which may be particularly beneficial to individuals with ASD [32]. Empirical evidence in education suggests that educational technology-based tools can foster enhanced motivation and facilitate the generalization of skills from the educational setting to different social contexts. However, findings from a number 
of interventions which have adopted a number of the game design principles have demonstrated success in improving this skill only to a limited degree [33]. Future research needs to be done in order to elucidate if this approach could be useful for children with ASD. Moreover, from our knowledge, there are no studies that propose the investigation of facial expression recognition from a developmental point of view including also a sample of individuals with ASD. Some recent reviews suggest that the performance in identifying emotions depends on the type of the assessment instrument, on the sample characteristics and if dynamic or static items are involved in the tasks used [34-35].

Based on the findings regarding the use of technological tools in the education process of children with ASD there was a series of computer-based interventions that were developed for this special population. The use of a screen can guide children with ASD to focus only on the relevant contents from the screen, helping them to ignore irrelevant data [36]. Considering the fact that children with ASD find social interactions challenging, mainly because are not predictable and intuitive, using a computer for providing the learning content instead of a teacher/therapist can make their job easier and more predictable [37]. Employing different types of technology-based tools for individuals with ASD may contribute to the development and practice of different abilities, which are necessary for social inclusion [38]. There are several programs that used different approaches in order to train the ability to recognize emotions, among them we mention: Mind reading Program [39]; The transporters [40, 41] and Reading the Mind in Films [42]. All these computer-based programs have tried to enrich the classical techniques of teaching emotions. Some of them used situation-based emotions illustrated in short films; others tried to gain attention by positioning the facial expressions on trains.

Although there are several limitations and unanswered questions in the domain of using technology-based instruments for children with ASD, such as to what extent they can generalize the information learned in a digitalized environment; these types of tools have a great potential of becoming standard educational programs. Therefore, is important to mention the advantages of using technological tools in the educational process of children with ASD. Correspondingly, users can remain motivated in the assessment task and intervention program because their interest can be controlled through personalized digital rewards; users can work at their level of understanding and these technological instruments do not require the use of social abilities that can be stressful for children with ASD [43-44].

\subsection{Objective of the Current Study}

In our study we propose to develop and test the effectiveness of a facial expression recognition instrument, which can be used both for typical developing individuals and for adolescents with ASDusing a technology-based paradigm. By including groups of children of different ages we also plan to approach the 
developmental aspect of emotion recognition process. Further, using different types of stimuli allows us to investigate if the ability to identify different emotions is dependent on the way the task is presented. The tasks used in our study ranged from full faces showing facial expression, to eyes part showing emotions to dynamic video stimuli that were presented in a tablet format. Therefore, our objective is to identify: a) if there are differences between the three types of the tests, with a better performance in the video phase compared to face and eye phases, b) if there are differences between the two groups investigated (typical developing children vs. children with ASD), c) if the ability of emotion recognition improves with age; and $d$. which type of emotion is better recognized in the two groups of participants.

\subsection{Description of the Emotions Proposed to Investigate in our Study}

For the development of our instrument, we have selected four emotions: happiness, sadness, anger and fear, which are known as basic emotional expressions and are recognized worldwide [45-48]. Every facial expression included in our study is easy to be reproduced and recognized by adults, mostly because they are able to identify the positions of facial muscles for each emotion. The elements that are involved in the production of each of the basic emotions are usually eyebrows, eyelids and lips [48]. Moreover, [49] each emotion has been associated with some action tendencies; some ideas that can be transmitted (communicated) through that emotion and some needs that can be expressed by using a type of expression or another (see Table 1).

Table 1

Description of action tendencies for the four basic emotions investigated in the study [49]

\begin{tabular}{|l|l|}
\hline \multicolumn{1}{|c|}{ Emotion } & \multicolumn{1}{c|}{ Behavioral and action tendencies } \\
\hline Anger & Aggression (verbal or physical/ direct or indirect) \\
\hline Fear & Avoiding threat / Seeking safety \\
\hline Happiness & Persevere in the same action \\
\hline Sadness & Withdrawal from enjoyable activities \\
\hline
\end{tabular}

\section{Methodology}

\subsection{Participants}

Before the implementation of the study the assessment protocol underwent an evaluation by the Ethics Committee from Babes-Bolyai University. All the participants' parents' were informed regarding the steps of the assessment 
protocol and each of them signed an informed consent through which they expressed their desire to participate at the study. Fifty-one children, aged 12-14 years, were enrolled in the study out of which 40 typical developing children and 11 children with ASD. Subjects from the typical developing group were recruited from Wesselenyi- Zalău Reformed High School Mass School. Children with ASD attend School for Inclusive Education in Simleul Silvaniei or Noro Center (a special daycare center which offers therapy services for children different developmental disorders). Children with ASD are aged 12-14 years and all have a confirmed diagnosis of ASD. Considering our hypothesis we have organized children in four different groups: one group of 12 years old children, one group of 13 years old children, one group of 14 years old children and one group of children with ASD (their ages varied from 12 to 14 years old).

\subsection{Procedure}

The setting for the experiments was a therapy room (about $20 \mathrm{~m} 2$ ) for the children with ASD, in which the children usually participated the therapeutic programs, so the children were familiar with the room and a regular classroom for the typically developing children. The therapist who performed the interaction is part of the research team of the Department of Special Education and she has experience in working with children with ASD. Children were presented in a tablet format with 12 stimuli illustrating facial expressions of the four basic emotions (happiness, anger, fear, and sadness). Children had to respond to the question, "How is the child feeling?" (see Figure 1)

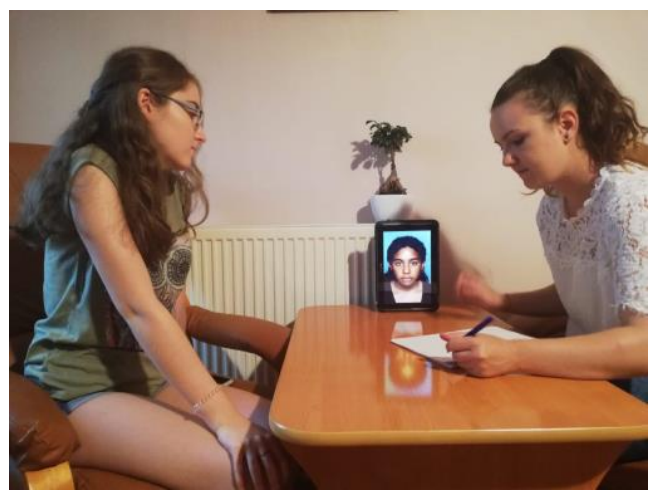

Figure 1

The experimental setting

\subsection{Instruments}

The test consisted in 12stimuli presented in a tablet format both for adolescents with ASD and for typical developing adolescents. The test had three phases, in each phase children were supposed to recognize the facial expressions. In the first 
two phases the children had to recognize the facial expressions from pictures showed on the tablet, the first 4 photos were illustrating faces with emotions and in the second phase the photos were illustrating eyes (see Figure 2). In order to make sure that the set of stimuli used are valid ones, we have used the stimuli developed in the Emotion and Development Branch in the National Institute of Mental Health (NIMH) Division of Intramural Research Programs. They have created Child Emotional Faces Picture Set (NIMH-ChEFS) which represent a collection of pictures illustrating adolescents that show different types of emotions.

For the third phase children had to recognize the emotions from a video, the video stimuli used were created especially for this study and illustrate the head and a small part of body images from an adolescent. In order to ensure the high quality of these videos, two specialists in clinical psychology have classified each stimuli. Afterwards we have used a basic validation procedure, in which the stimuli were presented to a sample of 30 students. The majority of them correctly identified the emotions, in this way we could be sure that the selected videos illustrate prototypes of the targeted emotions. The test was provided in a child-friendly format, it could be seen as attractive as a leisure activity as well as an educational tool.

Face phase

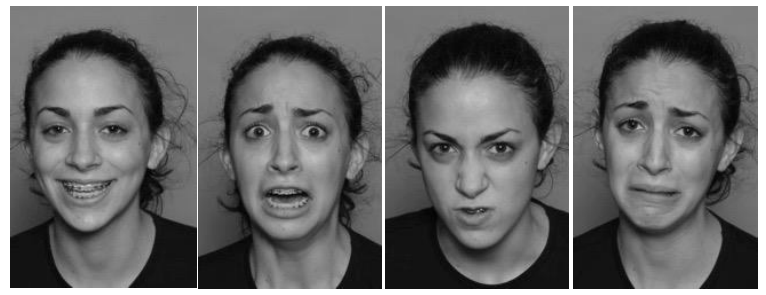

Eyes phase

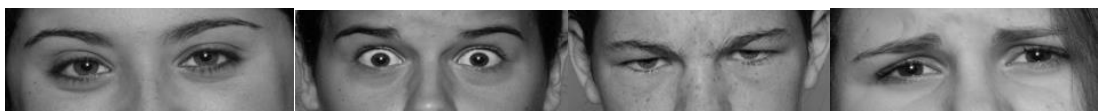

Video phase
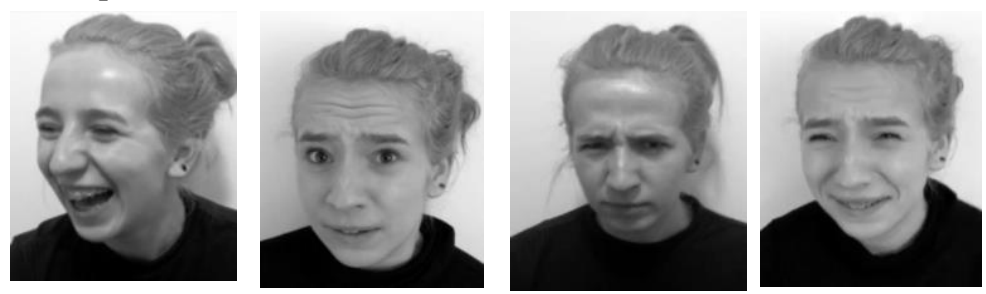

Figure 2

Examples of stimulus from each phase of the test 


\subsection{Results}

We have used the non-parametric test (Friedman) for comparing the performance in the 3 phases of the tests (face, eyes and video) for each type of group $(12,13$, 14 years old children and ASD children). The Friedman test represents an omnibus test, which is used to compare the results from two groups when the data is distributed differently than normal. This test is an alternative to performing the one-way ANOVA test. After the overall comparisons were performed in case of significant results Wilcoxon signed-rank test was used. By performing pairwise comparisons we could identify the conditions that yielded differences in performance that are statistically significant. The Wilcoxon signed-rank test is the nonparametric test equivalent to the dependent t-test.

For the 12 years old groupFriedman test showed significant differences between the three phases of the test, $C h i^{2}(2)=8.34 p<.015$. Pairwise comparisons showed that eye test phase yielded lower scores than video test $Z=-2.54, p=.011$. No significant differences were found between the face and eyes phases of the tests or between face and video phases of the test $p>.05$. Wilcoxon tests were used to investigate these differences (see Table 2)

In the 13 and 14 years old group we didn't find any significant differences between the three phases of the test $\mathrm{p}>.05$. (see Table 2)

In the ASD groupFriedman test showed significant differences between the three phases of the test, $C h i^{2}(2)=9.17 p=.01$. We found similar results as in the 12 years old groups, meaning that children yielded better performance in the video test phase compared to eyes test $\mathrm{Z}=-2.45, \mathrm{p}=.014$. No significant differences were found between the face and eyes phases of the tests or between the face and video phases of the test $p>.05$. (see Table 2)

Analysis per emotion tested

In the typical developing groups sadness had the highest rates of recognition in video phase of the tests. Therefore, in the group of children aged 14, all children recognized the emotion $(100 \%)$, it also had high rates of recognition in the group of children aged $13-83 \%$ and in the group of children aged $12-77 \%$.

Table 2

Means and standard deviations for the three phases (face, eyes and video) for each group

\begin{tabular}{|l|l|l|l|l|l|l|l|}
\hline & \multicolumn{2}{|l|}{ Face } & \multicolumn{2}{l|}{ Eyes } & \multicolumn{2}{l|}{ Video } \\
\cline { 2 - 7 } & $\mathrm{M}$ & SD & M & SD & M & SD \\
\hline 12 years old & 1.88 & 1.16 & 1.22 & 1.09 & 2.88 & 1.26 \\
\hline 13 years old & 2.25 & 0.98 & 1.87 & 1.03 & 2.25 & 1.26 \\
\hline 14 years old & 2.85 & 1.06 & 2.42 & 1.27 & 2.28 & 0.75 \\
\hline ASD group & 0.63 & 0.80 & 0.36 & 0.64 & 1.27 & 1.42 \\
\hline
\end{tabular}


In the typically developing group, after sadness, happiness and anger were also well identified by children, with rates higher than $50 \%$ in the group of children aged 13 and 14 in almost all phases of the test. In the group of children aged 12 sadness was best recognized in the face phase (88\%) and they had similar performances in the eyes and video phase (77\%). The same group had a good performance in recognizing anger in both three phases $(66 \%)$. In the typically developing group, fear was the least recognized emotion, in some cases (in the group of children aged 14) none of the participants identified correctly the emotion (see Figure 3,4 and 4).

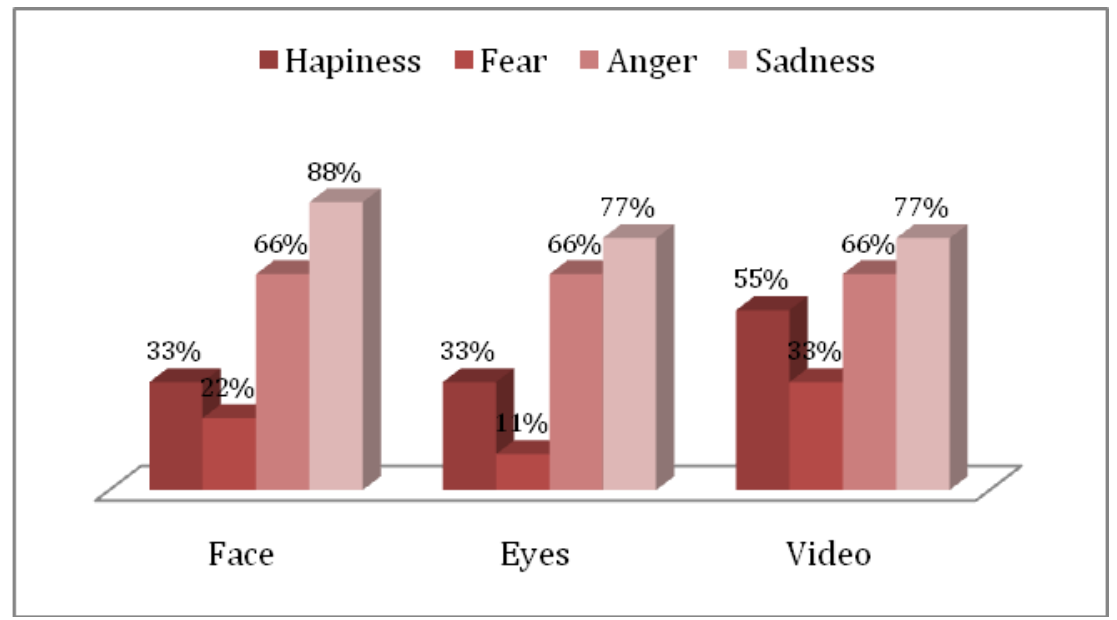

Figure 3

Performance of emotion recognition in the group of children aged 12 years - considering the emotion type

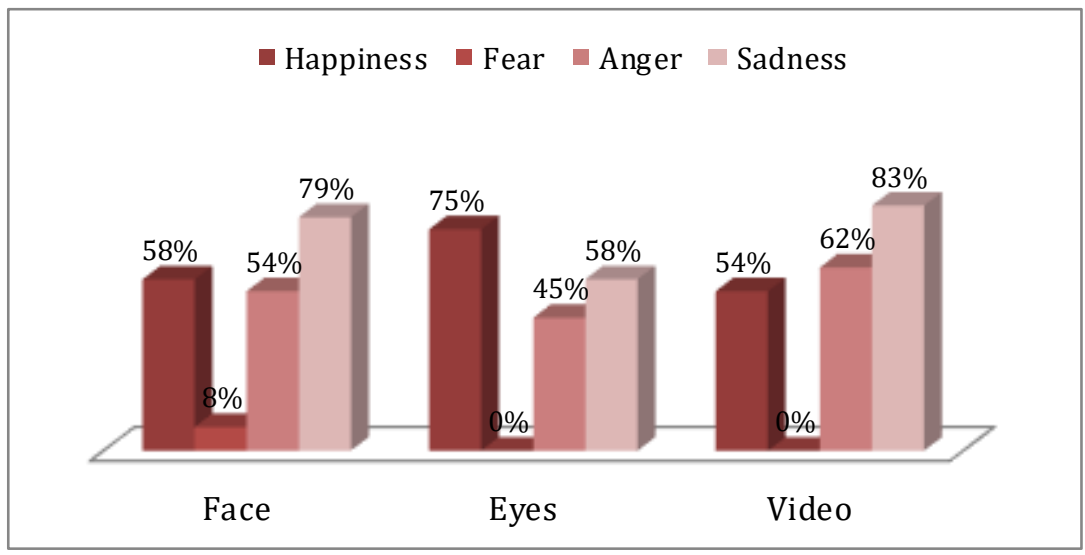

Figure 4

Performance of emotion recognition in the group of children aged 13 years - considering the emotion type 


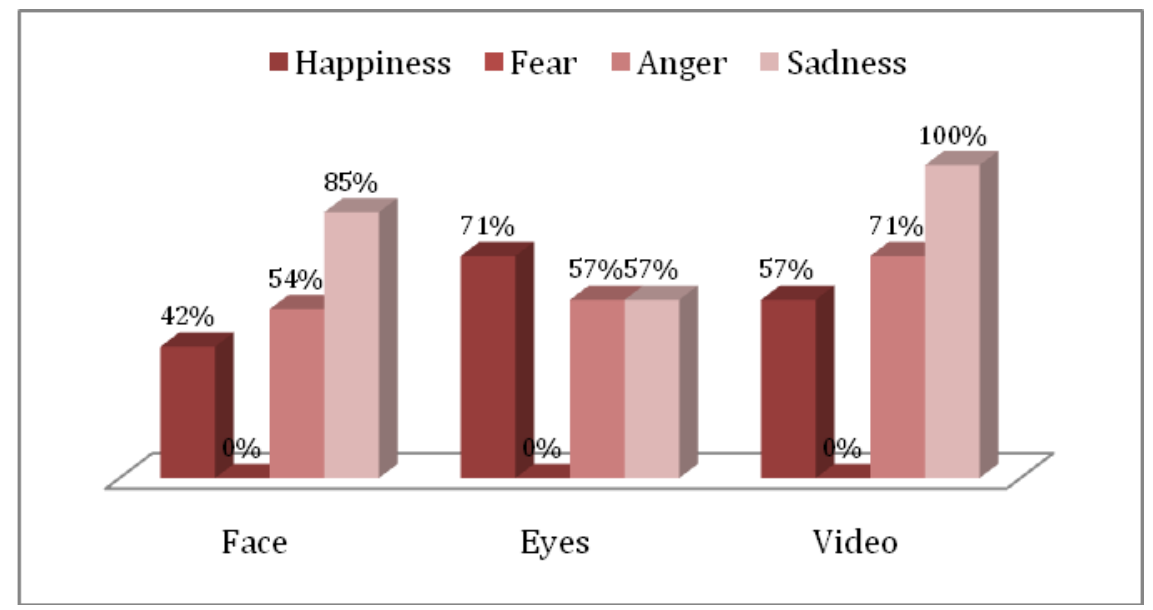

Figure 5

Performance of emotion recognition in the group of children aged 14 years - considering the emotion

type

For children with ASD happiness was the easiest emotion to recognize in the face phase $(36 \%)$ and in the video phase (63\%). In the eyes phase, children with ASD had serious difficulties in identifying emotions, even happiness was identified with a very low rate $(9 \%)$. In this phase, none of the children accurately identified fear and anger. Sadness was identified by children with ASD in the proportion of $27 \%$ in the video face, $18 \%$ in the eyes phase and $9 \%$ in the face phase. Similar to typical developing group, fear was the emotion most challenging for them to recognize. Also, they had some troubles identifying anger. Therefore, none of them recognized the fear in the eyes phase and it was correctly identified in the face phase only in a small percent $9 \%$ (see Figure 6).

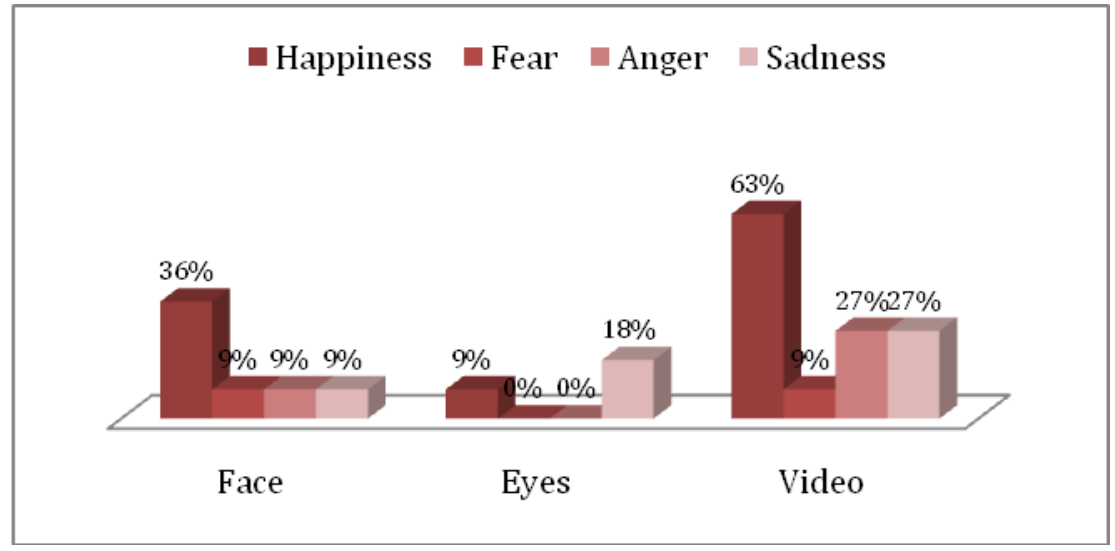

Figure 6

Performance of emotion recognition in the group of children with ASD - considering the emotion type 
Regarding the performance of the adolescence with ASD in general (regardless of the emotion presented) the results showed a great variability (see Table 3 ). None of the children reached the highest score and four of them didn't succeed in recognizing any of the facial expressions. Some possible explanations of their weak performance could be the difficulties in the task understanding, their cognitive impairment and their severity level on the spectrum.

Table 3

Performance of emotion recognition in the group of children with ASD

\begin{tabular}{|l|l|l|l|}
\hline & Video & Face & Eyes \\
\hline Participant 1 & 3 & 1 & 2 \\
\hline Participant 2 & 4 & 2 & 1 \\
\hline Participant 3 & 3 & 0 & 1 \\
\hline Participant 4 & 1 & 2 & 0 \\
\hline Participant 5 & 1 & 0 & 0 \\
\hline Participant 6 & 0 & 0 & 0 \\
\hline Participant 7 & 0 & 0 & 0 \\
\hline Participant 8 & 0 & 0 & 0 \\
\hline Participant 9 & 1 & 1 & 0 \\
\hline Participant 10 & 1 & 1 & 0 \\
\hline Participant 11 & 0 & 0 & 0 \\
\hline
\end{tabular}

\section{Discussions}

The competence of identifying facial expressions and using this information in a social environment represents an important step in the social inclusion [50-51]. Increased understanding of how typical and atypical children develop these abilities can generate effective assessment and intervention protocols which can enable individuals with ASD, and not only, to actively participate in the society. The findings of our study are in line with prior research [30] demonstrating that the type of stimuli that we use in order to identify emotions is important for the performance of children. Therefore, we have shown that the differences between the stimuli influence the way children with ASD recognized the emotions. They had a better performance in the video phase of the test compared to face phase and eyes phase of the test. We had similar findings for the 12 years old group of typically developing children. The fact that we didn't find statistical differences between the three phases of the test in the group of children aged 13 and 14 may be explained by the fact that as children grow they can better discriminate between different emotions regardless of the way in which they are presented to them. 
Regarding the differences in the types of emotions that children with ASD compared to typically developing children recognized better in the video phase we must be cautious when interpreting the main finding because of the small sample size. Therefore, even if the children with ASD better-recognized happiness (63\%) compared to sadness $(27 \%)$, the emotion that was the best recognized in a typically developing group, we cannot generalize these conclusions and assume that these differences may be observed in the general population.

\section{Conclusions}

The main finding of our work indicates that the instrument developed (the test with three phases) has a good potential to evaluate the abilities of recognizing facial expressions. Children really enjoyed using the tables during the study and we believe that the reason is because the test was simple and engaging. The novelty of this study consisted in the manner in which the test was applied and the use of dynamic stimuli in order to increase the accuracy in measuring emotion recognition. As we have mentioned also in the introduction part, there is a need of evidence-based technology-based tools in order to provide appropriate interventions for developing facial expression recognition especially for individuals with ASD. Our research tries to contribute to the international literature in the domain by developing a user-friendly instrument that can be used for the assessment of emotion recognition. Further research should extend the area of emotions investigated, from basic to complex emotions in a cross-cultural study. By increasing the understanding of how typical and atypical populations perceive (e.g. ASD, ADHD), label, express and use the information about facial expression in their social interaction, by using effective emotion regulation strategies, we can further develop intervention programs for improving individuals' quality of life [52], [53], [54].

The results may help to find the development directions of modern ICT based teaching methodologies in the field of Virtual or Augmented Reality supported learning [55] [56] [57] cooperative or project-based teaching [58], [59], [60] and other possibilities related to Cognitive Infocommunications supported education [61], [62], [63] [64] [65].

\section{Acknowledgment}

This research was supported by EFOP-3.6.1-16-2016-00003 grants, Establishment of long-term R \& D \& I process at the University of Dunaujvaros.

\section{References}

[1] Charles Darwin: The Expression of the Emotions in Man and Animals. Oxford: Oxford University Press; 1872

[2] Paul Ekman: Emotions Revealed. New York: Owl Books; 2003 
[3] Sherri Widen, James A. Russell:A closer look at preschoolers' freely produced labels for facial expressions, Developmental Psychology, Vol. 39, No. 1, 2003, pp. 114-128

[4] Karine Durand, Mathieu Gallay, Alix Seigneuri, Fabrice Robichon,JeanYves Baudouin: The development of facial emotion recognition: The role of configural information, Journal of Experimental Child Psychology, Vol. 97, No. 1, 2007, pp. 14-27

[5] Catherine J. Mondloch, Sybil Geldart, Daphne Maurer, Richard Le Grand: Developmental changes in face processing skills, Journal of Experimental Child Psychology, Vol. 86, No. 1, 2003, pp. 67-84

[6] De Sonneville, Verschoor, Njiokiktjien, Op het Veld, Toorenaar, Vranken: Facial identity and facial emotions: Speed, accuracy, and processing strategies in children and adults, Journal of Clinical and Experimental Neuropsychology, Vol. 24, No. 2, 2002, pp. 200-213

[7] Thomas LA, De Bellis MD, Graham R, LaBar KS. Development of emotional facial recognition in late childhood and adolescence. Developmental Science, Vol. 10, No. 5, 2007, pp. 547-558

[8] Jennifer L. Lindner, Lee A. Rosen: Decoding of emotion through facial expression, prosody, and verbal content in children and adolescents with Asperger's syndrome, Journal of Autism and Developmental Disorders., Vol. 36, No. 6, 2006, pp. 769-777

[9] Celani Giorgio, Marco Walter Battacchi, Letizia Arcidiacono: The understanding of the emotional meaning of facial expressions in people with autism, Journal of Autism and Developmental Disorders, Vol. 29. No. 1, pp. 29:57-66

[10] MirkoUljarevic, and Antonia Hamilton: Recognition of emotions in autism: a formal meta-analysis, Journal of Autism and Developmental Disorders, Vol. 43, No. 7, 2013, pp. 1517-26

[11] OferGolan, Yana Sinai-Gavrilov, Simon Baron-Cohen: The Cambridge mindreading face-voice battery for children (CAM-C): complex emotion recognition in children with and without autism spectrum conditions, Molecular Autism, Vol. 6, No. 1, 2015, pp. 1-22

[12] Margaret Hudepohl, Diana Robins, Tricia King, Christopher Henrich: The role of emotion perception in adaptive functioning of people with autism spectrum disorders, Autism, Vol. 19, No. 1, 2015, pp. 107-112

[13] Jeannette M. Haviland, Mary Lelwica: The induced affect response: 10week-old infants' responses to three emotion expressions, Vol. 23, No. 1, 1987, pp. 97-104 
[14] Jukka Leppänen, Margaret C. Moulson, Vanessa K. Vogel-Farley,Charles A. Nelson:An ERP study of emotional face processing in the adult and infant brain, Child Development, Vol. 78, No. 1, 2007, pp. 232-425

[15] Alin Klin, Warren Jones, Robert Schultz, Fred Volkmar, Donald Cohen: Visual fixation patterns during viewing of naturalistic social situations as predictors of social competence in individuals with autism, Archives of general psychiatry, Vol. 59, No. 9, 2002, pp. 809-16

[16] Geraldine Dawson, Sara Jane Webb, James McPartland: Understanding the nature of face processing impairment in autism: insights from behavioral and electrophysiological studies, Developmental Neuropsychology, Vol. 27, No. 3, 2005, pp. 403-424

[17] Eleni A. Papagiannopoulou, Kate M. Chitty, Daniel F. Hermens, Ian B. Hickie, Jim Lagopoulos: A systematic review and meta-analysis of eyetracking studies in children with autism spectrum disorders, Social Neuroscience, Vol. 9, No. 6, 2014, pp. 610-632

[18] Quetin Guillon, Bernadette Rogé, Mohammad H. Afzali, Sophie Baduel, Jeanne Kruck, Nouchine Hadjikhani: Intact perception but abnormal orientation towards face-like objects in young children with ASD, Scientific Reports, Vol. 6, No. 1, 2016, p. 22119

[19] Simon Baron-Cohen, Sally Wheelwright, Therese Jolliffe: Is there a "Language of the Eyes"? Evidence from normal adults, and adults with autism or asperger syndrome. Vis cogn., Vol. 4, No. 3, 1997, pp. 311-331

[20] Kevin A. Pelphrey, Noah J. Sasson, Steven J. Reznick, Gregory Paul, Barbara Goldman, Joseph Piven: Visual scanning of faces in autism, Journal of Autism and Developmental Disorders, Vol. 32, No. 4, pp. 249261

[21] Steve Berggren, Ann-Charlotte Engström, Sven Bölte: Facial affect recognition in autism, ADHD and typical development, CognitiveNeuropsychiatry, Vol. 21, No. 3, pp. 213-227

[22] Francesc Happé: Autism: cognitive deficit or cognitive style? Trends in Cognitive Science,Vol. 3, No. 6, 1999, pp. 216-222

[23] Christine Deruelle, Cecilie Rondan, Bruno Gepner, Carole Tardif: Spatial frequency and face processing in children with autism and Asperger syndrome, Journal of Autism and Developmental Disorders, Vol. 34, No. 2, 2004, pp. 199-210

[24] Joshua J. Diehl, Loisa Bennetto, Duane Watson, Christine Gunlogson, Joyce McDonough: Resolving ambiguity: a psycholinguistic approach to understanding prosody processing in high-functioning autism, Brain Language., Vol. 106, No. 2, 2008, pp. 144-52 
[25] Ruth B. Grossman, Helen Tager-Flusberg: Reading faces for information about words and emotions in adolescents with autism, Research in Autism Spectrum Disorders. Vol. 2, No. 4, 2008, pp. 681-695

[26] Philip, Whalley, Stanfield, Sprengelmeyer, Santos, Young, Atkinson, Calder, Johnstone, Lawrie, Hall: Deficits in facial, body movement and vocal emotional processing in autism spectrum disorders, Psychological Medicine, Vol. 4, No. 11, 2010, pp. 1919-1929

[27] James B. Grossman, Ami Klin, Alice S. Carter, Fred Volkmar: Verbal bias in recognition of facial emotions in children with Asperger syndrome. The Journal of Child Psychology and Psychiatry and Allied Disciplines, Vol. 41, No. 3, 2000, pp. 369-379

[28] Fulvia Castelli: Understanding emotions from standardized facial expressions in autism and normal development, Autism, Vol. 9, No. 4, 2005, pp. 428-449

[29] Delphine B. Rosset,Cecilie Rondan, David Da Fonseca, Andreia Santos, Brigitte Assouline, Christine Deruelle: Typical emotion processing for cartoon but not for real faces in children with autistic spectrum disorders, Journal of Autism and Developmental Disorders, Vol. 38, No. 5, 2008, pp. 919-925

[30] Patricia Howlin, Anna Moore: Diagnosis in autism: A survey of over 1200 patients in the UK, Autism, Vol. 1, No. 2, 1997, pp. 135-162

[31] Simon Baron-Cohen, Alan M. Leslie, Uta Frith: Does the autistic child have a "theory of mind"? Cognition, Vol. 21, No. 1, 1985, pp. 37-46

[32] Elisabeth M. Whyte, Joshua M. Smyth, K. Suzanne Scherf: Designing serious game interventions for individuals with autism, Journal of Autism and Developmental Disorders, Vol. 45, No. 12, 2015, pp. 3820-3831

[33] James W. Tanaka, Julie M. Wolf, Cheryl Klaiman, Kathleen Koenig, Jeffrey Cockburn, Lauren Herlihy, Carla Brown, Sherin Stahl, Martha Kaiser, Robert Schultz: Using computerized games to teach face recognition skills to children with autism spectrum disorder: the Let's Face It! Program, Journal of Child Psychology and Psychiatry, Vol. 51, No. 8, 2010, pp. 944-952

[34] Zara Ambadar, Jonathan W. Schooler, Jeffrey F. Cohn: Deciphering the enigmatic face: The importance of facial dynamics in interpreting subtle facial expressions, Psychological Science,Vol. 16, No. 5, 2005, pp. 403-410

[35] Jessica Tracy, Richard Robins, Roberta Schriber, Marjorie Solomon: Is emotion recognition impaired in individuals with autism spectrum disorders? Journal of Autism and Developmental Disorders, Vol. 41, No. 1, 2011, pp: 102-9 
[36] Miriam Silver, Peter Oakes: Evaluation of a new computer intervention to teach people with autism or Asperger syndrome to recognize and predict emotions in others, Autism, Vol. 5, No. 3, 2001, pp. 299-316

[37] Cristina Anamaria Pop, Ramona Simut, Sebastian Pintea, Jelle Saldien, Alina Rusu, Daniel David, Johan Vanderfaeillie, Dirk Lefeber, Bram Vanderborght: Can the social robot Probo help children with autism to identify situation-based emotions? A series of single case experiments. International Journal of Humanoid Robotics, Vol. 10, No. 3, 2013, pp. 1350025

[38] Orit E. Hetzroni, Juman Tannous: Effects of a computer-based intervention program on the communicative functions of children with autism, Journal of Autism and Developmental Disorders, Vol. 34, No. 2, 2004, pp. 95-113

[39] Simon Baron-Cohen: Mind reading: the interactive guide to emotionsversion 1.3." Jessica Kingsley, London, 2007

[40] Ofer Golan, Emma Ashwin, Yael Granader, Suzy McClintock, Kate Day, Victoria Leggett, Simon Baron-Cohen: Enhancing emotion recognition in children with autism spectrum conditions: An intervention using animated vehicles with real emotional faces, Journal of Autism and Developmental Disorders, Vol. 40, No. 3, 2010, pp. 269-279

[41] Simon Baron-Cohen, Ofer M. Golan, and Emma JM Ashwin: Teaching emotion recognition to children with autism spectrum conditions,BJEP Monograph Series II, Number 8-Educational Neuroscience. British Psychological Society Vol. 115, No. 127, 2012, pp. 115-127

[42] Golan Ofer, Simon Baron-Cohen, Yael Golan: The 'reading the mind in films' task [child version]: Complex emotion and mental state recognition in children with and without autism spectrum conditions, Journal of Autism and Developmental Disorders, Vol. 48, No. 8, 2008, pp. 1534-1541

[43] David Moore, Paul McGrath, John Thorpe: Computer-aided learning for people with autism-a framework for research and development,Innovations in education and training international, Vol. 37, No. 3, 2000, pp. 218-228

[44] Sarah Parsons, Peter Mitchell: The potential of virtual reality in social skills training for people with autistic spectrum disorders, Journal of Intellectual Disability Research, Vol. 46, No. 5, 2002, pp. 430-443

[45] Ernst Huber: Evolution of facial musculature and facial expression, 1931

[46] Irenaus Eibl-Eibesfeldt: Human ethology. Routledge, 2017

[47] Carroll E. Izard: The face of emotion, 1971

[48] Paul Ekman, Wallace V. Friesen. "Unmasking the face Englewood Cliffs." Spectrum-Prentice Hall, New Jersey, 1975 
[49] Christina G. Kohler, Travis Turner,Neal M. Stolar, Warren B. Bilker, Collen M. Brensinger, Raquel E. Gur, Ruben C. Gur: Differences in facial expressions of four universal emotions, Psychiatry Research, Vol. 128, No. 3, 2004, pp. 235-244

[50] Madeline B. Harms, Alex Martin, Gregory L.Wallace: Facial emotion recognition in autism spectrum disorders: a review of behavioral and neuroimaging studies, Neuropsychol Review, Vol. 20, No. 3, 2010, pp. 290-322

[51] Amy G. Halberstadt, Susanne A. Denham, Julie C. Dunsmore: Affective social competence, Social development, Vol. 10, No. 1, 2001, pp. 79-119

[52] Andreea Robe, Anca Dobrean, Ioana Cristea, Costina R.Pasarelu, Elena Predescu: Atttention-deficit/hyperactivity disorder and task-related heart rate variability: A systematic review and meta-analysis, Neuroscience and Biobehavioral Reviews, Vol. 99, 2019, pp. 11-22

[53] Shimrit Fridenson-Hayo, Steve Berggren, Amandine Lassalle, Shahar Tal, Delia Pigat, Sven Bölte, Simon Baron-Cohen, Ofer Golan: Basic and complex emotion recognition in children with autism: cross-cultural findings, Molecular Autism, Vol. 7, 2016, pp. 1-52

[54] Cristina Costescu, et al.: Assessing Visual Attention in Children Using GP3 Eye Tracker, Proceedings of the $10^{\text {th }}$ IEEE International Conference on Cognitive Infocommunications (CogInfoCom), Naples, 2019, pp. 343-348

[55] Ildikó Horváth: Evolution of teaching roles and tasks in VR/AR-based education, Proceedings of the $9^{\text {th }}$ IEEE International Conference on Cognitive Infocommunications (CogInfoCom), Budapest, 2018, pp. 355360

[56] Laszlo Bognar, Eva Fancsikne, Peter Horvath, Antal Joos, Balint Nagy, Gyorgyi Strauber: Improved learning environment for calculus courses, Journal of Applied Technical and Educational Sciences, Vol. 8, No. 4, 2018, pp. 35-43

[57] Adam Csapo, Ildiko Horvath, Peter Galambos and Peter Baranyi: VR as a Medium of Communication: from Memory Palaces to Comprehensive Memory Management, Proceedings of the $9^{\text {th }}$ IEEE International Conference on Cognitive Infocommunications (CogInfoCom), Budapest, 2018, pp. 389-394

[58] Ildiko Horvath: Innovative engineering education in the cooperative VR environment, Proceedings of the $7^{\text {th }}$ IEEE Conference on Cognitive Infocommunications (CogInfoCom), Wrocław, 2016, pp. 359-364

[59] Ilona Heldal, Carsten Helgesen: The Digital HealthLab: Supporting Interdisciplinary Projects in Engineering and in Health Education, Journal of Applied Technical and Educational Sciences, Vol. 8, No. 4, 2018, pp. 421 
[60] Robert Pinter, SanjaMaravicCisar: Measuring Team Member Performance in Project Based Learning, Journal of Applied Technical and Educational Sciences, Vol. 8, No. 4, 2018, pp. 22-34

[61] Peter Baranyi, Adam Csapo, Gyula Sallai, Cognitive Infocommunications (CogInfoCom), Springer International Publishing Switzerland, 2015, p. 219

[62] Elod Gogh et al.: Metacognition and Lifelong Learning, $9^{\text {th }}$ IEEE International Conference on Cognitive Infocommunications, 2018, pp. 271276

[63] Cristina Costescu and Adrian Rosan: Development an assessment protocol to identify the characteristics of ASD using eye-tracking for special education purpose, Journal of Applied Technical and Educational Sciences, Vol. 9, No. 4, 2019, pp. 70-87

[64] Gergely Sziladi et al.: The analysis of hand gesture based cursor position control during solve an IT related task, $8^{\text {th }}$ IEEE International Conference on Cognitive Infocommunications, 2017, pp. 413-418

[65] E. Gogh, A. Kovari, Experiences of Self-regulated Learning in a Vocational Secondary School, Journal of Applied Technical and Educational Sciences, Vol. 9, No. 2, 2019, pp. 72-86 